\title{
POLYMERIC MICRO-GRIPPER FOR APPLYING MECHANICAL STIMULATION ON THREE-DIMENSIONAL CELL AGGREGATES
}

\author{
Q. Wang, S. Zhao, J.K. Choi, X. He, and Y. Zhao ${ }^{*}$ \\ Department of Biomedical Engineering, The Ohio State University, Ohio, USA
}

\begin{abstract}
This work reports the development of a polymeric micromechanical device to provide mechanical stimulation to three-dimensional (3D) cell aggregates that are a few hundred of $\mu \mathrm{m}$ in size. Different from current loading technologies for $3 \mathrm{D}$ cultured cells, the device allows for the application of individually adjustable mechanical stimulation to a number of $3 \mathrm{D}$ cell aggregates, thus allowing parallel operation for in-depth investigation of loading parameter-dependent cell responses. Proof-of-concept experiments showed that the unique strain pattern generated by this device is able to guide the differentiation of embryonic stem cells towards a specific direction without the use of chemical inducing factors. This work indicates that $3 \mathrm{D}$ mechanical stimuli can be a promising inducing factor for regulating stem cell differentiation.
\end{abstract}

\section{INTRODUCTION}

Mechanical signal is universal in living tissues and organisms and is critical in regulating various physiological behaviors. The role of these mechanical signals on cell fate is often investigated by loading on in vitro cultured cell monolayers by engineered devices where the extracellular microenvironment is well controllable and the cells are shielded from undesired stimuli. The most prevalent method is to seed the cells on a substrate, and generate surface strain by deforming the substrate. Most commonly, an elastomeric membrane serves as the substrate. The membrane deformation can be induced by stretching the membrane directly by mechanical actuators or indirectly by deforming the connected elastomer structures using vacuum. Despite of its research popularity, the use of adherent cell monolayer for studying cell responses to mechanical signals is debatable due to the fact that the two-dimensional (2D) cell monolayer confines cell adhesion within a planar or a curved surface, in sharp contrast to those in natural tissues where the cells are allowed to reach out to a three-dimensional (3D) space. As such, for a cell in a $2 \mathrm{D}$ monolayer, nearly $50 \%$ of its surface is exposed to an artificial engineering substrate, while only a very small portion is in contact with other cells. This is substantially different from the natural environment where the entire surface area of a cell is often in contact with other cells through extracellular matrix. The lack of 3D structural architecture may lead to a totally different pattern of intracellular structures, intercellular mechanotransduction, signaling and in turn affect the overall cell function. Although the mechanism governing the difference between 2D and 3D cell culturing is still poorly understood, and whether the $3 \mathrm{D}$ culturing environments are superior over the 2D counterparts is arguable, there is a general consensus that the difference in morphological characteristics due to different culturing environment is likely to induce functional dissimilarities. This is backed up by many reports showing that $3 \mathrm{D}$ cultured cells by various methods exhibit distinct behaviors from the corresponding 2D models.

When the cells are cultured in 3D environments, the mechanical stimulation applied towards the cells is no longer inplane. Current technologies for providing mechanical stimulation to $3 \mathrm{D}$ cultured cells include the use of a platen to press on cellseeded hydrogels, electrospun matrix, or other 3D culturing substrates, stretching cell-seeded ring-shaped collagen gels to apply tensile strain; loading on microfluidic channels which is filled with cell-seeded collagen gel, just to name a few [7-11]. All these 3D loading methods have shown their effectiveness in applying tensile or compressive mechanical strain to cells (i.e. cellembedded constructs or embryoid bodies). Nonetheless, the strain pattern that can be delivered is limited: in most cases only uniaxial strain can be applied. Numerous studies in 2D cell loading have reported that the cell response is often dependent on the strain pattern, e.g. uni-axial strain and equi-biaxial strain can result in very different effects on the mechanical regulation of stem cells [12]. It is plausible to infer that such effect also is valid for 3D loading, i.e. the response of 3D cultured cells exposed to uni-axial strain loading is different from those exposed to other strain pattern types. Moreover, since cell response to mechanical stimulation is often dependent on the loading parameters, parallel experimentation with simultaneous loading on multiple 3D samples is preferred.

In this work, we designed and fabricated a polymeric microdevice to apply compressive strain to an array of nine $3 \mathrm{D}$ cultured cell aggregates. Different from previous uni-axial loading, the cell aggregates were simultaneously loaded at four different spots, leading to a more complex strain distribution. The results showed that the device can successfully apply strains on alginate microbeads and cell-loaded microcapsules. Proof-of-concept cell loading test showed that 72-hour cyclic loading with the unique strain pattern delivered by this device was able to increase the expression of cardiac troponin I (cTnI) in the aggregates of undifferentiated R1 murine embryonic stem (ES) cells without the help of specific chemical inducing factors.

\section{MATERIALS AND METHODS \\ Device Design}

The device consists of multiple loading sites which can be individually actuated to simultaneously apply a differential pattern of mechanical compressive strains to a number of 3D cell aggregates. Each loading site consists of four microscale pillar-like structures on top of a thin circular membrane, and a microfluidic channel placed underneath (Figure 1a\&b). The four pillars were arranged axially symmetrically on the circular membrane and their distance to the membrane center was adjusted to gain an enclosed space with the same diameter of the $3 \mathrm{D}$ cell aggregate to be loaded. Once the pressure in the microfluidic channel changes, a differential pressure occurs across the membrane and deforms the membrane, thus titling the pillars and changing the volume of space enclosed by them. In particular, once a positive pressure is applied, the circular membrane deflects upwards and the volume of the enclosed space increases (Figure 1c). The increased space is designed to be slightly greater than the size of the 3D cell aggregate so as for easy loading. Once the positive pressure is removed, the pillars returned to their vertical standings to hold the $3 \mathrm{D}$ cell aggregate in position. Once a negative pressure is applied, the circular membrane deflects downwards and the volume of the enclosed space decreases (Figure 1d). The four pillars thus press on the surface of the cell aggregate to apply mechanical stimulation. The strain magnitude and profile are functions of the applied differential pressure. To ensure good contact between the pillars and the cell aggregate and repeatability of the loading 

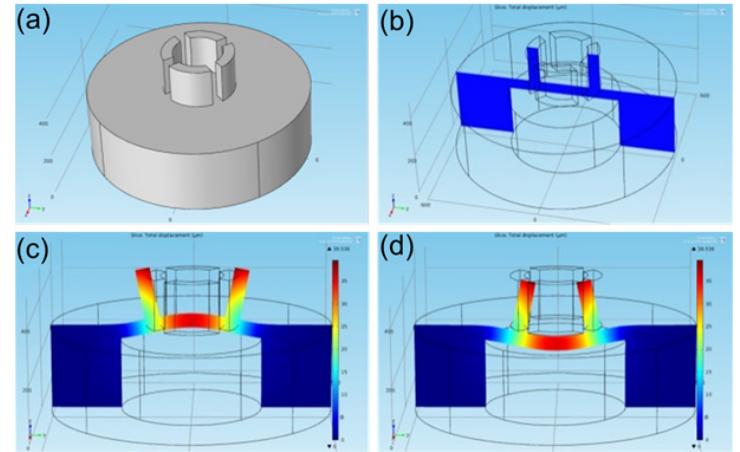

Figure 1: Schematic of the micro-gripper and the working principle of mechanical stimulation: (a) a micro-gripper consists of four fingers enclosing a cylindrical space; (b) cross-sectional view showing the micro-gripper on the top of a circular thin membrane. The micro-gripper deforms upon (c) a positive differential pressure to increase the enclosed volume or (d) a negative differential pressure to decrease the enclosed volume.

parameters, each pillar has a curved inner surface to match the surface of the spherical cell aggregate. The size of the circular membrane was determined to have the pillars gain the maximal tilting angle at a given pressure. The four-pillar assembly is referred to a micro-gripper and each pillar to a micro-gripper finger hereafter.

Given that the 3D cell aggregates used in this study has an average diameter of $225 \mu \mathrm{m}$, the inner diameter of the cylindrical space enclosed by the four fingers was set as $225 \mu \mathrm{m}$. The height of the fingers was determined in consideration of actuation range and operation reliability: if a finger is too tall, the tips of the four fingers may meet each other before a desired strain can be applied. If a finger is too short, the spherical cell aggregate tends to be unstable upon compression and has a high risk of escaping from the enclosed space especially under a large strain magnitude. After a trial-and-error experimental test assisted by finite element analysis, the height of the micro-gripper fingers was determined to be $180 \mu \mathrm{m}$ for the cell aggregates with $225 \mu \mathrm{m}$ in diameter. Each finger was $50 \mu \mathrm{m}$ thick (the distance from the outer surface of the finger to its inner surface). Finite element analysis showed that with such a width and a height-to-width ratio, the micro-gripper finger itself does not yield significant deformation while pressing on the cell aggregate. According to the plate theory, the slope of deflection in a thin circular membrane along the radial direction reaches the maximal in the region $0.3 \mathrm{R}$ to $0.7 \mathrm{R}$ distant to the membrane center, where $\mathrm{R}$ is the membrane radius. The radius of the circular membrane was thus determined as $500 \mu \mathrm{m}$ in diameter. The thickness was determined as $55 \mu \mathrm{m}$.

\section{Device fabrication}

The entire device was made of polydimethylsiloxane (PDMS). The thin circular membrane with micro-grippers was fabricated using a double-side replica molding process [13], in which the upper mold for creating the micro-gripper fingers and lower mold for creating the circular thin membrane were prepared separately (Figure 2). For the upper mold fabrication, extruded micro-gripper fingers $(225 \mu \mathrm{m}$ in inner diameter, $325 \mu \mathrm{m}$ in outside diameter, and $180 \mu \mathrm{m}$ in height; the gap between the adjacent fingers was $50 \mu \mathrm{m}$ ) were first created by patterning SU-8 negative photoresist (SU8 2100, Microchem, MA) on a silicon wafer by photolithography. PDMS prepolymer (10:1 w/w ratio) was dispensed on the wafer, cured and peeled off, forming complementary features of micro-gripper fingers in the PDMS

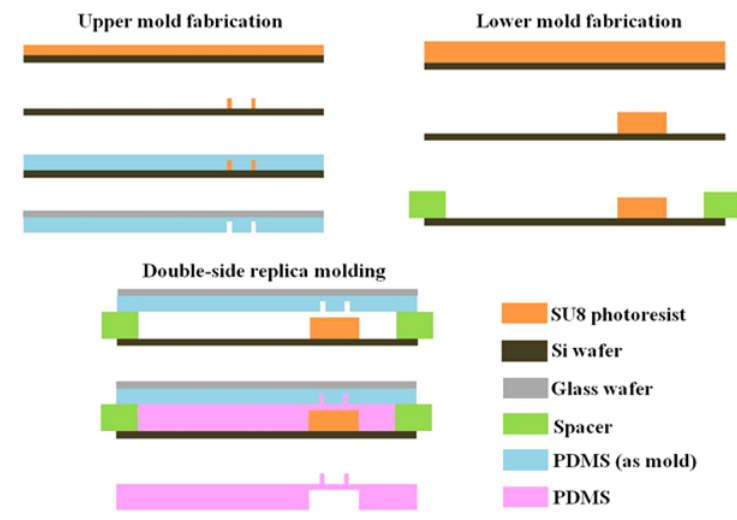

Figure 2: Fabrication process of the loading component. The PDMS substrate carrying the micro-gripper and the circular loading membrane was fabricated by double-side replica molding.

substrate. The outmost surface of the PDMS substrate was then treated with $0.1 \%$ Hydroxypropyl Methycellulose (HPMC) aqueous solution for 30 minutes in order to form a separation layer for easing subsequent mold releasing. For the lower mold fabrication, SU-8 microposts $(500 \mu \mathrm{m}$ in diameter and $245 \mu \mathrm{m}$ in height) were patterned on a silicon wafer. Afterwards, the upper and lower molds were placed face-to-face, aligned and separated by spacers that were $300 \mu \mathrm{m}$ thick. This left a $55 \mu \mathrm{m}$ gap between the top surfaces of the upper and lower molds. While holding the two molds in position, PDMS prepolymer (10:1 w/w ratio) was filled into the gap, cured, and released from the two molds. The 55 $\mu \mathrm{m}$ thick PDMS circular membranes with micro-gripper fingers on their top surfaces were thus obtained, as shown in Figure 3.

After device fabrication, deionized water was pumped into the microfluidic channels underneath the membrane using a syringe pump for generating a hydraulic differential pressure across the loading membrane. The use of hydraulic instead of pneumatic actuation is in observation of possible air leaking and long-term strain drifting due to the gas permeability of PDMS. Once the channels are fully filled with water, the cell culturing surface of the device was cleaned by $1 \%$ Sodium dodecyl sulfate (SDS) solution for 1 hour, followed by rinsing with $70 \%$ ethanol, and exposed to UV light overnight. After sterilization, the cell culturing surface was rinsed twice with phosphate buffered saline (PBS), each for 5 minutes.

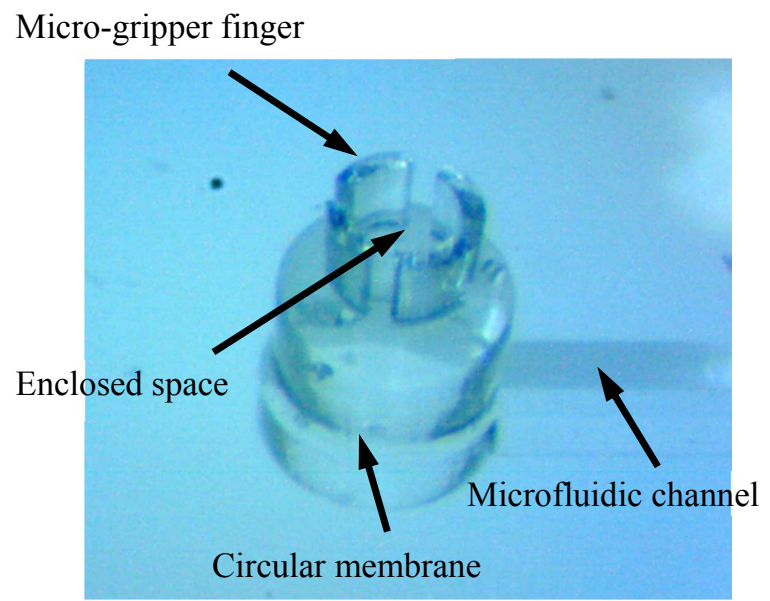

Figure 3: Optical micrograph showing a micro-gripper with four fingers and the underlying membrane. 
In this study, microdevices with an array of nine microgrippers were fabricated for mechanical stimulation testing. Each micro-gripper was connected with an individual microfluidic channel and can thus be actuated independently.

\section{Preparation of Solid Alginate Microbeads and Cell-Loaded Microcapsules}

Solid alginate beads was prepared by electrospray using a previously reported method [14]. In brief, 2.0\% (w/v) sodium alginate (Sigma) dissolved in $250 \mathrm{mM}$ mannitol solution was pumped through a 30 gauge syringe needle at the flow rate of 27 $\mu \mathrm{l} / \mathrm{min}$ and sprayed into $100 \mathrm{mM}$ calcium chloride as the gelling bath under a $1.8 \mathrm{kV}$ open electric field. The solid alginate beads with the average diameter of $225 \mu \mathrm{m}$ were then collected from the gelling solution and loaded to the devices.

R1 murine embryonic stem (ES) cells from ATCC were cultured in ES medium with Knockout ${ }^{\circledR}$ DMEM supplemented, $15 \%$ Knockout $^{\circledR}$ serum, $1000 \mathrm{U} / \mathrm{ml} \mathrm{LIF}, 4 \mathrm{mM}$ l-glutamine, $0.1 \mathrm{M}$ 2-mercaptoethanol, $10 \mu \mathrm{g} / \mathrm{ml}$ gentamicin, $100 \mathrm{U} / \mathrm{ml}$ penicillin, and $100 \mu \mathrm{g} / \mathrm{ml}$ streptomycin in gelatin coated tissue culture flasks with medium being changed daily. For the microcapsules loaded with cells, coaxial electrospray design was used to generate microcapsules with aqueous liquid core and alginate (hydrogel) shell, which is preferred for the embryonic cells to form single uniform aggregates. In general, the core solution containing $5 \times 10^{6}$ per $\mathrm{ml} \mathrm{ES} \mathrm{cells} \mathrm{and} 1 \%(\mathrm{w} / \mathrm{v})$ sodium carboxymethyl cellulose in $250 \mathrm{mM}$ mannitol solution and the shell solution of $2.0 \%$ alginate (w/v) were pumped through the concentric inner $(28 \mathrm{G})$ and outer lumen $(21 \mathrm{G})$ of a customized syringe needle respectively. Under the open electric field $(\sim 2.1 \mathrm{kV})$, the core solution and the shell solution were sprayed into the gelling solution $\left(100 \mathrm{mM} \mathrm{CaCl}_{2}\right)$ at $40 \mu \mathrm{l} / \mathrm{min}$ and $60 \mu \mathrm{l} / \mathrm{min}$, respectively. The encapsulated ES cells were then collected, washed with $0.5 \mathrm{M}$ mannitol, and cultured in ES medium for 7 days, until spherical aggregates were formed in the liquid core of core-shell microcapsules.

\section{Mechanical Stimulation and Immunostaining}

Cyclic loading with the maximal differential pressure of -13 $\mathrm{kPa}$ was applied at $0.5 \mathrm{~Hz}$ for 72 hours. The un-loaded cell-loaded microcapsules were used at the control group. Both groups were maintained in regular DMEM with $10 \% \mathrm{FBS}, 100 \mathrm{U} / \mathrm{ml}$ penicillin, and $100 \mathrm{mg} / \mathrm{l}$ streptomycin for the entire period of mechanical stimulation.

After mechanical stimulation, cTnI was examined by immunostainning. Encapsulated cell aggregates were collected and then released from capsules by incubating them in $75 \mathrm{mM}$ sodium citrate for 1 minute. Afterwards, cell aggregates were fixed by $4 \%$ PFA for 15 minutes, washed by PBS for three times and incubated in $3 \%$ bovine serum albumin (BSA) for 30 minutes to block nonspecific binding at room temperature. The primary antibody of cTnI and corresponding secondary antibody were then applied. Before imaging, the nuclei of cell aggregates were further stained with $5 \mu \mathrm{M}$ Hoechst 33342 for 15 minutes. The images were taken using an Olympus FV1000 confocal microscope and the intensity of each condition was analyzed using ImageJ (NIH).

\section{RESULTS AND DISCUSSIONS}

The solid alginate microbeads and ES cell-loaded microcapsules (both were $225 \mu \mathrm{m}$ in diameters) were transferred into the micro-grippers, followed by cyclic application of negative differential pressure by the syringe pump. The deformation of the micro-gripper fingers and that of the solid alginate microbeads (or ES cell-loaded microcapsules) were measured using optical
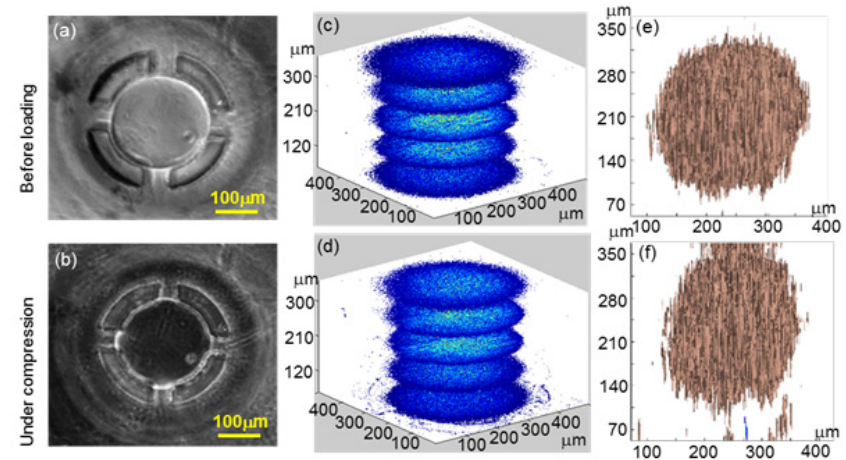

Figure 4. Deformation of solid alginate microbeads before and under mechanical stimulation: $(a \& b)$ phase contrast images; $(c \& d)$ z-stack fluorescent images of the microbead; (e\&f) the $3 D$ profile of the microbead reconstructed from the fluorescence images. The loading pressure was $-13 \mathrm{kPa}$. The microbeads were with $2 \%$ alginate concentration.

microscopy. Z-stack phase contrast images were obtained to show the boundaries of the microbeads as they deformed. A sequence of 90 images with $4 \mu \mathrm{m}$ step distance was captured under each loading condition by scanning the microsphere from bottom to top. The image at the mid-plane of the microsphere was selected for deformation analyses, where the mid-plane before compression was determined from the image with the largest diameter; the corresponding mid-plane under compression was determined from its relative position to the top and the bottom of the micro-gripper fingers, assuming there was no slip between the microsphere and the micro-gripper fingers during mechanical stimulation.

\section{Mechanical Loading on Solid Microbeads}

The measurement in solid alginate microbeads showed that the deformation of all the microbeads increased with the differential pressure, while the microbeads with a higher alginate concentration yields a smaller deformation compared to that with a lower alginate concentration. Specifically, for microbeads with $1.2 \%$ alginate concentration, the mean diameter change at the midplane was $5.0 \mu \mathrm{m}$ at $-2.1 \mathrm{kPa}$. This number increased to $28.3 \mu \mathrm{m}$ as the differential pressure changed to $-13.0 \mathrm{kPa}$. For microbeads with $2.0 \%$ alginate concentration, the mean diameter change at the midplane was $3.5 \mu \mathrm{m}$ at $-2.1 \mathrm{kPa}$, and $20.7 \mu \mathrm{m}$ at $-13.0 \mathrm{kPa}$. For microbeads with $3.0 \%$ alginate concentration, the mean diameter change at the mid-plane was by $1.1 \mu \mathrm{m}$ at $-2.1 \mathrm{kPa}$ and $5.4 \mu \mathrm{m}$ at $13.0 \mathrm{kPa}$. The dependence of the diameter change with the alginate concentration is believed due to the different Young's moduli of the microbeads, which affects the maximal allowable strain range. FITC-dextran labelling was used to visualize the deformation of the microbeads. Z-stack phase contrast images were obtained to show the boundaries of the microbeads as they deformed. A representative deformation profile before and under compression was shown in Figure 4, with the alginate concentration of $2 \%$.

\section{Mechanical Loading on Cell-Loaded Microcapsules}

The deformation of the microcapsules upon loading was also measured. The results showed that both the entire microcapsule and the cell core exhibited discernable deformation (Figure 5). For instance, for the microcapsules with the shell of $2.0 \%$ alginate concentration, the mid-plane of the entire microcapsule exhibited the mean diameter change of $3.7 \mu \mathrm{m}$ at $-2.1 \mathrm{kPa}$, and $23.4 \mu \mathrm{m}$ at $13.0 \mathrm{kPa}$, and the mid-plane of the cell core exhibited the mean diameter change of $3.0 \mu \mathrm{m}$ at $-2.1 \mathrm{kPa}$, and $20.2 \mu \mathrm{m}$ at $-13.0 \mathrm{kPa}$. It was noted that the deformation of cell-loaded microcapsules 

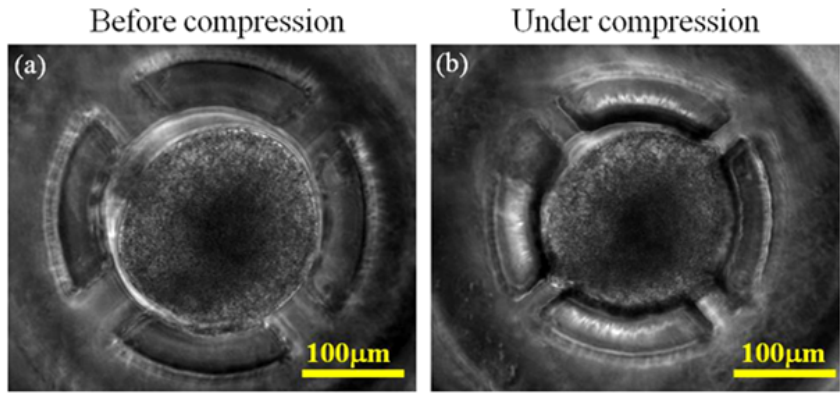

Figure 5: Deformation of a microcapsule (2\% alginate concentration, with ES cell aggregates) upon loading: (a) microcapsule before compression; (b) microcapsule under compression. The differential pressure was $-13.0 \mathrm{kPa}$.

were greater than that of the solid microbeads at the same alginate concentration. This indicated that the Young's modulus of the cell aggregates is lower than the alginate shell with $2 \%$ alginate concentration.

\section{Mechanical Stimulation Regulated Stem Cell Differentiation}

Immunostaining result after 72 hours of loading showed that the mechanical stimulation group exhibited significantly higher expression of $\mathrm{cTnI}$ comparing to those in the control group (Figure 6 ). This indicated that cyclic mechanical stimulation by the microgrippers can promote the differentiation of 3D ES cell aggregates into cardiac like phenotypes without the help of chemical inducing factors. This may open up a new avenue for tailoring the multidirectional differentiation of ES cells. More systematical investigation of the relationship between the loading parameters, the strain pattern, and ES cell differentiation is underway.
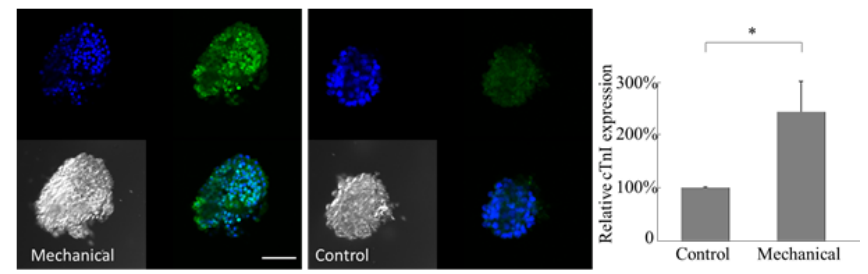

Figure 6: Immunostaining of cTnI (green) in ES cell aggregates, Hoechst staining of cell nuclei (blue), and differential interference contrast (DIC) images showing the aggregates morphology. Scale bar: $100 \mu \mathrm{m}$. *: Statistically significant $(p<0.05)$.

\section{CONCLUSIONS}

In this study, we develop a microdevice with an array of micro-grippers to apply mechanical strain towards 3D cell microaggregates encapsulated within an alginate shell. The results showed that the device can successfully load on both solid microbeads and cell-loaded microcapsules. The mechanical stimulation elevated the expression of cTnI, a cardiac marker, indicating the mechanical strain generated by the micro-grippers may regulate the differentiation of ES cells a specific phenotype. The microdevice also shows its compatibility with parallel operation, which is essential for investigating loading parameterdependent cell responses to mechanical signals.

\section{REFERENCES}

[1] H. Lu, L. Y. Koo, W. M. Wang, D. A. Lauffenburger, L. G. Griffith, and K. F. Jensen, "Microfluidic shear devices for quantitative analysis of cell adhesion," Analytical Chemistry, vol. 76, pp. 5257-5264, 2011/10/17 2004.
[2] N. Watanabe, H. Kataoka, T. Yasuda, and S. Takatani, "Dynamic Deformation and Recovery Response of Red Blood Cells to a Cyclically Reversing Shear Flow: Effects of Frequency of Cyclically Reversing Shear Flow and Shear Stress Level," Biophysical journal, vol. 91, pp. 1984-1998, 2006.

[3] G. Keller, "Embryonic stem cell differentiation: emergence of a new era in biology and medicine," Genes \& Development, vol. 19, pp. 1129-1155, 2005.

[4] M. Hata, K. Naruse, S. Ozawa, Y. Kobayashi, N. Nakamura, N. Kojima, et al., "Mechanical Stretch Increases the Proliferation While Inhibiting the Osteogenic Differentiation in Dental Pulp Stem Cells," Tissue Engineering Part A., vol. 19, pp. 625-633, 2013.

[5] S. Saha, L. Ji, J. J. de Pablo, and S. P. Palecek, "Inhibition of human embryonic stem cell differentiation by mechanical strain," Journal of Cellular Physiology, vol. 206, pp. 126-137, 2006.

[6] E. T. Pineda, R. M. Nerem, and T. Ahsan, "Differentiation patterns of embryonic stem cells in two- versus threedimensional culture," Cells, tissues, organs, vol. 197, pp. 399410, 2013.

[7] A. H. Huang, M. J. Farrell, M. Kim, and R. L. Mauck, "Longterm dynamic loading improves the mechanical properties of chondrogenic mesenchymal stem cell-laden hydrogel.," European Cells and Materials, vol. 19, pp. 72-85, 2010.

[8] K. L. Au-yeung, K. Y. Sze, M. H. Sham, and B. P. Chan, "Development of a Micromanipulator-Based Loading Device for Mechanoregulation Study of Human Mesenchymal Stem Cells in Three-Dimensional Collagen Constructs," Tissue Engineering Part C: Methods. , vol. 16, pp. 93-107, 2010.

[9] V. F. Shimko and W. C. Claycomb, "Effect of Mechanical Loading on Three-Dimensional Cultures of Embryonic Stem Cell-Derived Cardiomyocytes," Tissue Engineering Part A., vol. 14, pp. 49-58, 2008.

[10] C.-r. Wan, S. Chung, and R. Kamm, "Differentiation of Embryonic Stem Cells into Cardiomyocytes in a Compliant Microfluidic System," Annals of Biomedical Engineering, vol. 39, pp. 1840-1847, 2011.

[11] C. Moraes, G. Wang, Y. Sun, and C. A. Simmons, "A microfabricated platform for high-throughput unconfined compression of micropatterned biomaterial arrays," Biomaterials, vol. 31, pp. 577-584, 2010.

[12] J. S. Park, J. S. F. Chu, C. Cheng, F. Chen, D. Chen, and S. $\mathrm{Li}$, "Differential effects of equiaxial and uniaxial strain on mesenchymal stem cells," Biotechnology and Bioengineering, vol. 88, pp. 359-368, 2004.

[13] Q. Wang, X. Zhang, and Y. Zhao, "Micromechanical stimulator for localized cell loading: fabrication and strain analysis," Journal of Micromechanics and Microengineering, vol. 23, p. 015002, 2013.

[14] W. Zhang, S. Zhao, W. Rao, J. Snyder, J. K. Choi, J. Wang, et al., "A novel core-shell microcapsule for encapsulation and 3D culture of embryonic stem cells," Journal of Materials Chemistry B, vol. 1, pp. 1002-1009, 2013.

[15] Q. Wang, X. Zhang, and Y. Zhao, "A microscale mechanical stimulator for generating identical in-plane surface strains toward live cells on multiple loading sites," Sensors and Actuators B: Chemical, vol. 194, pp. 484-491, 2014.

\section{CONTACT}

*Y. Zhao, tel: +1-614-247-7424; zhao.178@,osu.edu 